\title{
Serum lipids and associated factors of dyslipidemia in the adult population in Shenzhen
}

\author{
Wen-Qing Ni, Xiao-Li Liu, Zhi-Peng Zhuo, Xue-Li Yuan, Jin-Ping Song, Hong-Shan Chi and Jian Xu*
}

\begin{abstract}
Background: Dyslipidemia is one of the most important independent modifiable risk factors for cardiovascular diseases. The objective of this study was to determine the prevalence and risk factors for dyslipidemia in Shenzhen, a special economic zone and large metropolitan area neighboring Hong Kong.
\end{abstract}

Methods: A cross-sectional survey of 1,995 adults with a mean age of 46.56 years was conducted between February and July 2011 using a multistage stratified cluster random sampling. All the subjects were administered questionnaires regarding socio-demographic characteristics and other possible factors associated with the prevalence of dyslipidemia. Fasting venous blood samples were collected to assess the lipid profile. Weight, height, waist circumference, and blood pressure were measured.

Results: The mean concentrations of total cholesterol (TC), triglycerides (TG), high density lipoprotein cholesterol (HDL-C), and low density lipoprotein cholesterol (LDL-C) were $5.11 \pm 1.15 \mathrm{mmol} / \mathrm{L}, 1.59 \pm 1.47 \mathrm{mmol} / \mathrm{L}, 1.42 \pm 0.33 \mathrm{mmol} / \mathrm{L}$, and $3.22 \pm 0.84 \mathrm{mmol} / \mathrm{L}$, respectively. High values of TC, TG, low HDL-C, and high LDL-C were obtained in $14.49 \%, 16.14 \%, 8.82 \%$, and $12.13 \%$ of the 1,995 participants, respectively. The prevalence of dyslipidemia was $34.64 \%$, among which $25.04 \%$ of subjects were aware. Presence of dyslipidemia was significantly associated with increasing age, smoking status, hypertension, diabetes, and body mass index.

Conclusions: High prevalence of dyslipidemia with relative low awareness in Shenzhen was found. A comprehensive strategy is required for the prevention, screening, treatment, and control of dyslipidemia in Shenzhen.

Keywords: Dyslipidemia, Risk factors, Serum lipid, Prevalence

\section{Introduction}

Dyslipidemia is defined as the derangements of one or more of the lipoproteins in blood, such as elevated in total cholesterol (TC), low density lipoprotein cholesterol (LDL-C) and/or triglycerides (TG), or low levels of high density lipoprotein cholesterol (HDL-C) alone [1]. The results of other studies suggested that many persons with dyslipidemia were overtly under-diagnosed and it is estimated that prevalent cases of dyslipidemia in the nine major countries will increase at the rate of $1.76 \%$ per year to surpass 500 million in 2022 [2]. The number of people with dyslipidemia is expected to reach 78 million in the major countries by 2022, of which, one sixth of all cases will occurre in China [2]. The prevalence of dyslipidemia is high and increases continuously in many

\footnotetext{
* Correspondence: anniexu73@126.com

Shenzhen Center for Chronic Disease Control, No.2021, Buxin Rd., Shenzhen, Guangdong 518020, P.R. China
}

developing countries as a result of the westernization of diet, obesity, aging of population, reduced physical activity, and other adverse lifestyle changes [3]. Dyslipidemia is one of the most important and modifiable risk factors for cardiovascular diseases, which is also a major cause of morbidity and leads to mortality worldwide [4]. The morbidity and mortality of cardiovascular diseases in China are expected to increase both in absolute number and as a proportion of total disease burden, parallel changes in lifestyle and diet in the next 20 years [5].

Several clinical trials have indicated that the treatment of dyslipidemia is effective in both primary and secondary prevention of cardiovascular events [6, 7]. Treatment of dyslipidemia can reduce the risk of heart disease by approximately $30 \%$ over a 5 -year period [8]. Nevertheless, surveys in China have showed poor awareness, treatment and control of dyslipidemia [9, 10]. In order to improve dyslipidemia control, it is critical to understand 
the characteristics of those who have dyslipidemia. Therefore, new initiatives and resources can be targeted and tailored to the needs of high-risk populations. Studies conducted in different regions of China have measured relationships between potential risk factors and dyslipidemia, however, differences in socioeconomic status, demographic composition, lifestyle, dietary, alcohol intake, and physical activity in Shenzhen suggest the necessity of reevaluating these factors in Shenzhen's current context [11, 12]. Therefore, the primary goal of present study was to investigate the prevalent status of dyslipidemia in a representative sample of the urban population aged more than 20 years that includes 12 streets from 8 districts of the city of Shenzhen, and its association with other risk factors.

\section{Materials and methods Sampling sites}

As China's first Special Economic Zone to spur economic growth after the near collapse of the socialist centrally-planned economy in 1978, Shenzhen has transformed from an agriculture-based Baoan County into a $21^{\text {st }}$ century metropolis housing over millions of people. Unprecedented social and economic development in Shenzhen has led to changes in patterns of diet, physical activity, and other socio-demographic characteristics that are contributing to large increases in the prevalence of cardiovascular disease and other chronic diseases [13]. Dyslipidemia is a well known risk factor for cardiovascular disease. A prevention measure against the epidemic of dyslipidemia will undoubtedly push down the morbidity and mortality of cardiovascular diseases. The associated factors of dyslipidemia in Beijing, Shaoxing, and Delhi has have been evaluated; however, little data are available in Shenzhen [11, 12, 14].

The present study was conducted as a part of the China Health and Nutrition Survey at the Center for Chronic Disease Control of Shenzhen. In brief, the survey used a multistage stratified cluster random sampling method to select a representative sample of permanent residents in 8 different districts in Shenzhen city. The first stage of sampling was stratified by district and population distribution on the basis of Shenzhen population data from 2010, which involved the random selection of 12 streets from those 8 districts. In the second stage, one residential community was randomly selected from each selected street. In the third stage, about 75 households were randomly selected from the residential communities selected in the earlier stage. In the last stage, eligible family member from each designated household was chosen.

\section{Study population}

Volunteers were recruited from selected households from February 2011 to July 2011. The eligibility criteria were set as following: aged 20 years or above, living in Shenzhen for more than 5 years, men or non-pregnant women, confirmed not to present with cancer, severe liver disease, pancreatitis, polycystic ovarian syndrome, systemic lupus erythematosus, and kidney diseases.

From February 2011 to July 2011, 1,995 participants were recruited according to the eligibility criteria. All participants received information and publicity about the health risk that may result from dyslipidemia exposure. All participants have the chances to receive the health check and biochemical determination results if they provided their telephone number or other contact information. The study received ethnicity approval of the Center for Chronic Disease Control of Shenzhen. Written informed consent was obtained from all participants before the study.

\section{Questionnaire survey}

Structured questionnaires were administered to collect information on potential risk factors for elevated blood lipids, as well as possible factors associated with dyslipidemia. Volunteers were interviewed in person 1 to 2 hours after blood collection and each questionnaire took approximately $20 \mathrm{~min}$ to complete. Questions covered general information (age, gender, ethnic, place of residence, employment and marital status), socioeconomic status (household per capita income and education level), health behaviors (drinking habit, level of physical activity, and smoking habit), and personal medical history.

The age groups were classified according to results from Shenzhen 2010 census as 20 to 29 years, 30 to 39 years, 40 to 49 years, 50 to 59 years, 60 to 69 years, 69 to 79 years, and $\geq 80$ years. Employment status was classified into one of the followings: homemaker, employed, unemployed, retired, and others. Marital status was categorized as single, married, widowed, and divorced. Educational level was categorized into 5 groups according to the number of years of education $(0,1-6,7-$ $9,10-12$, and $\geq 12$ years). Subjects were asked to choose 1 of 10 income categories that best represented the household per capita income for the past 12 months: $<5,000$ RMB; 5,000 to 9,999 RMB; 10,000 to 14,999 RMB; 15,000 to 19,999 RMB; 20,000 to 24,999 RMB; 25,000 to 29,999 RMB; 30,000 to $34,999 \mathrm{RMB} ; 35,000$ to $39,999 \mathrm{RMB} ; \geq$ 40,000 RMB; and unclear. The questions regarding smoking, which are described in details elsewhere, permit stratification of participants into categories such as ever-smokers (including: current smokers and ex-smokers) and never smokers [15]. Participants were also defined as habitual drinker (drink once per day or more), nonhabitual drinker (six times per week to once per month), or non-drinker (almost never) [16]. In the survey, the term "moderate to vigorous intensity physical activity" was 
defined as physical activity causing at least some sweating and shortness of breath, whereas the term "light physical activity" was defined as causing no sweating or shortness of breath [17]. Furthermore, physical activity was defined as moderate to vigorous intensity physical activity not less than once a week.

\section{Physical examination}

Anthropometric examinations were administered in the morning after fasting overnight, followed by body measurements, which were taken by trained examiners using a standardized protocol. Height, body weight, waist circumference, and blood pressure of all participants were recorded. Height and weight were measured with the participants wearing light dress without shoes using analog scales. Waist circumference (WC) was measured at the midpoint level of midaxillary line between the 12th rib head and the superior anterior iliac spine, with measuring time at the end of normal expiration. Body mass index (BMI) was calculated by dividing body weight (in kilograms) by the square meters of height. Blood pressure was measured using a mercury sphygmomanometer on the right arm of each of the participants in a comfortable sitting position after a $30 \mathrm{~min}$ rest period. Three consecutive blood pressure measurements were performed, and the mean of these three measurements was applied in the subsequent analysis.

\section{Blood sample collection and analyses}

Vein blood samples were collected after 10 to 14 hours of fasting. Each glass automatic hemostix was marked with the volunteer's identification code and placed into ice in a portable refrigerator before transporting to laboratory. In our laboratory, the blood samples were separated by centrifugation at $1000 \times \mathrm{g}$ for $15 \mathrm{~min}$ at $4{ }^{\circ} \mathrm{C}$, and then supernatant serum was collected. Serum glucose (fasting blood glucose), TC, TG, LDL-C, and HDL$\mathrm{C}$ concentrations were measured by using commercially available kits (Olympus System Reagents, Olympus Diagnostica $\mathrm{GmbH}$, Clare, Ireland) by autoanalyzer (Olympus AU400 System, Olympus, Tokyo, Japan). Serum TG were measured using the glycerol-3 phosphate oxidase-phenol + 4-aminophenazone enzymatic colorimetric method and TC by the cholesterol oxidase method. HDL-C was estimated in a series of reaction steps using an enzymatic cholesterol reagent after precipitation of non-HDL-C with magnesium/dextran, and LDL-C was estimated using a third-generation direct LDL cholesterol assay. The laboratory variation coefficient of TC, TG, HDL-C, and LDL-C was $1.5 \%, 4.3 \%, 3.0 \%$, and $3.9 \%$, respectively (indicating that long term standardization of TC, TG, HDL-C and LDL-C can be obtained with automated enzymatic methods utilizing commercially available reagents). Furthermore, as measures of quality control and assurance, analytical duplicates, and reagent blanks were routinely included in the analytical sequence for detection of precision and signs of contamination, respectively. In all cases, analyses were performed after the correct values had been confirmed by the instrument. All blood samples were analyzed within 4 hours after being collected.

\section{Definitions of dyslipidemia, obesity, diabetes and hypertension}

Dyslipidemias were defined according to Chinese criteria [18]. High TC was defined as a TC value $\geq 6.22 \mathrm{mmol} / \mathrm{L}$ and hypertriglyceridemia (high TG) was defined as a serum TG level $\geq 2.26 \mathrm{mmol} / \mathrm{L}$. Low HDL-C was defined as a serum HDL-C level $<1.04 \mathrm{mmol} / \mathrm{L}$. High LDL-C was defined as a serum LDL-C level $\geq 4.14 \mathrm{mmol} / \mathrm{L}$. In the present study, high TC, and/or high LDL-C, and/or low HDL-C and/or high TG, and/or having a history of dyslipidemia disease in the past year, and/or currently receiving treatment with lipid-lowering medications was regarded as dyslipidemia. Awareness of dyslipidemia was defined as answering "yes" to the following question: "During the past year, has a healthcare professional told that you had dyslipidemia?".

Body mass index (BMI) was used as a measure of body size. Participants with $\mathrm{BMI}<18.5 \mathrm{~kg} / \mathrm{m}^{2}$ were classified as underweight, those with BMI of 18.5 to $23.9 \mathrm{~kg} / \mathrm{m}^{2}$ as normal weight, those with BMI of 24 to $27.9 \mathrm{~kg} / \mathrm{m}^{2}$ as overweight, and those with BMI of $\geq 28 \mathrm{~kg} / \mathrm{m}^{2}$ as obese [19]. Two sets of cut offs were used to define central obesity in terms of waist circumference. These were $>95 \mathrm{~cm}$ for men and $>90 \mathrm{~cm}$ for women on the basis of specific guidelines for central obesity [19].

Hypertension was defined as mean systolic blood pressure $\geq 140 \mathrm{mmHg}$ and/or diastolic blood pressure $\geq 90 \mathrm{mmHg}$, and/or self-reported antihypertension treatment in the last 2 weeks. Diabetes was defined as fasting blood glucose (FBG) $\geq 7.0 \mathrm{mmol} / \mathrm{L}$, and/or selfreported treatment of diabetes with antidiabetes medication in the previous 2 weeks.

\section{Statistical analyses}

The mean and standard deviation of continuous variables were expressed as mean $\pm \mathrm{SD}$, and percentage was calculated for categorical variables. Age and other anthropometric examination characteristic differences between groups defined by gender were evaluated using Student's $t$-test. The chi-squared test or Fisher's exact test was used to determine significant differences in proportions among categorical variables. Normal distribution of the serum lipid concentration was tested by Kolmogorov-Smirnov test, and showed a highly skewed. Changes in outcome measures between the different groups not following normal distribution were tested by 
Mann-Whitney $U$ test or Kruskal-Wallis $\mathrm{H}$ test for independent variables.

The univariate and multivariate logistic regression models were used to investigate the probability to having dyslipidemia according to socio-demographic and other variables. Statistical analysis of the data was performed by Statistical Package for Social Sciences (SPSS) version 21.0 software (SPSS Inc., Chicago, IL, USA). A $P$ value of $<0.05$ was considered to be statistically significant. We estimated age standardized prevalence rate, and the standard population used in the direct method was the Shenzhen 2010 census population.

\section{Results}

\section{Socio-demographic and other characteristics of} participants

The Socio-demographic and other characteristics of the participants are summarized in Table 1 . Of the 1,995 participants, $44.36 \%(n=885)$ were men, $55.64 \%(n=$

Table 1 Socio-demographic and other characteristics of participants in the study

\begin{tabular}{|c|c|c|c|c|c|}
\hline Characteristics & General $(n=1995)$ & Women $(n=1110)$ & Men $(n=885)$ & Statistics & $P$ \\
\hline Age (years) & $46.56 \pm 15.26$ & $46.07 \pm 15.05$ & $47.17 \pm 15.53$ & $t=1.594$ & 0.111 \\
\hline BMI $\left(\mathrm{Kg} / \mathrm{m}^{2}\right)$ & $23.52 \pm 3.49$ & $23.21 \pm 3.47$ & $23.89 \pm 3.46$ & $t=4.320$ & $<0.001$ \\
\hline SBP $(\mathrm{mm} \mathrm{Hg})$ & $115.65 \pm 17.58$ & $113.92 \pm 18.31$ & $117.81 \pm 16.36$ & $t=4.993$ & $<0.001$ \\
\hline $\mathrm{DBP}(\mathrm{mm} \mathrm{Hg})$ & $74.97 \pm 10.11$ & $73.16 \pm 10.06$ & $77.24 \pm 9.71$ & $t=9.140$ & $<0.001$ \\
\hline WC $(\mathrm{cm})$ & $82.04 \pm 10.51$ & $79.16 \pm 10.14$ & $85.67 \pm 9.83$ & $t=14.438$ & $<0.001$ \\
\hline FBG $(\mathrm{mmol} / \mathrm{L})$ & $5.39 \pm 1.32$ & $5.28 \pm 1.13$ & $5.52 \pm 1.52$ & $t=3.937$ & $<0.001$ \\
\hline \multicolumn{6}{|l|}{ Physical activity, n (\%) } \\
\hline Yes & $1591(79.75)$ & 979 (88.19) & $612(69.15)$ & \multirow[t]{2}{*}{$x^{2}=110.599$} & \multirow[t]{2}{*}{$<0.001$} \\
\hline No & $404(20.25)$ & $131(11.81)$ & $273(30.85)$ & & \\
\hline \multicolumn{6}{|l|}{ Smoking status, n (\%) } \\
\hline Ever smoker & $414(20.75)$ & $10(0.90)$ & $404(45.65)$ & \multirow[t]{2}{*}{$x^{2}=599.570$} & \multirow[t]{2}{*}{$<0.001$} \\
\hline Never smoker & $1581(79.25)$ & $1100(99.10)$ & $481(54.35)$ & & \\
\hline \multicolumn{6}{|l|}{ Drinking habit, n (\%) } \\
\hline Non-drinker & $1295(64.91)$ & $887(79.91)$ & $408(46.10)$ & \multirow[t]{3}{*}{$x^{2}=248.308$} & \multirow[t]{3}{*}{$<0.001$} \\
\hline Non-habitual drinker & $586(29.37)$ & $192(17.30)$ & $394(44.52)$ & & \\
\hline Habitual drinker & $114(5.72)$ & $31(2.79)$ & $83(9.38)$ & & \\
\hline \multicolumn{6}{|l|}{ Marital status, n (\%) } \\
\hline Single & $129(6.47)$ & $69(6.22)$ & $60(6.78)$ & \multirow[t]{4}{*}{$x^{2}=30.648$} & \multirow[t]{4}{*}{$<0.001$} \\
\hline Married & $1771(88.77)$ & $962(86.67)$ & 809 (91.41) & & \\
\hline Widowed & $25(1.25)$ & $21(1.89)$ & $4(0.45)$ & & \\
\hline Divorced & $70(3.51)$ & $58(5.22)$ & $12(1.36)$ & & \\
\hline \multicolumn{6}{|l|}{ Educational level, n (\%) } \\
\hline 0 year & $73(3.66)$ & $63(5.68)$ & $10(1.13)$ & \multirow[t]{5}{*}{$x^{2}=65.626$} & \multirow[t]{5}{*}{$<0.001$} \\
\hline $1-6$ years & $261(13.08)$ & $172(15.50)$ & 89 (10.06) & & \\
\hline $7-9$ years & $524(26.26)$ & $286(25.77)$ & $238(26.89)$ & & \\
\hline 10-12 years & $855(42.86)$ & $475(42.79)$ & $380(42.94)$ & & \\
\hline$\geq 12$ years & $282(14.14)$ & $114(10.26)$ & 168 (18.98) & & \\
\hline \multicolumn{6}{|l|}{ Employment status, n (\%) } \\
\hline Unemployed & $92(4.61)$ & $56(5.05)$ & $36(4.07)$ & \multirow[t]{5}{*}{$x^{2}=184.955$} & \multirow[t]{5}{*}{$<0.001$} \\
\hline Retired & $487(24.41)$ & $262(23.60)$ & $225(25.42)$ & & \\
\hline Others & $156(7.82)$ & $77(6.94)$ & 79 (8.93) & & \\
\hline Homemaker & $297(14.89)$ & $269(24.23)$ & $28(3.16)$ & & \\
\hline Employed & $963(48.27)$ & $446(40.18)$ & $517(58.42)$ & & \\
\hline
\end{tabular}

Data are expressed as mean \pm SD if not indicated.

$B M I$ Body mass index, FBG Fasting blood glucose, WC Waist circumference 
1110) were women, the mean age was $46.56 \pm 15.26$ (20-96) years, $83.26 \%$ attained a junior high school or higher education, $88.77 \%$ were married, and $48.27 \%$ were employed. The rate of smoking was $20.75 \%$, and drinking was $35.09 \%$, and $79.75 \%$ of the study subjects reported having regular physical activities. In terms of anthropometric, the mean levels of average BMI, SBP, DBP, WC and FBG for all 1,995 participants were $23.52 \pm 3.49 \mathrm{Kg} / \mathrm{m}^{2}, 115.65 \pm 17.58 \mathrm{~mm} \mathrm{Hg}, 74.97 \pm$ $10.11 \mathrm{~mm} \mathrm{Hg}, 82.04 \pm 10.51 \mathrm{~cm}$, and $5.39 \pm 1.32 \mathrm{mmol} /$ $\mathrm{L}$, respectively.

Significant differences were found in different gender groups for BMI, SBP, DBP, WC, FBG, physical activity, smoking status, drinking habit, marital status, educational level, and employment status (Table 1). Subjects were examined for the presence of diabetes and hypertension. Approximately $8.78 \%$ of participants suffered from diabetes and one fifth of subjects $(22.31 \%)$ suffering from hypertension (Table 2). Using BMI as the anthropometric indicator, the prevalence of overweight and obesity among the 1,995 participants were $32.03 \%$ and $10.33 \%$, respectively (Table 2 ).

\section{Serum lipid concentrations}

The mean concentrations of TC, TG, HDL-C, and LDL-C were $5.11 \pm 1.15 \mathrm{mmol} / \mathrm{L}, 1.59 \pm 1.47 \mathrm{mmol} / \mathrm{L}$, $1.42 \pm 0.33 \mathrm{mmol} / \mathrm{L}$, and $3.22 \pm 0.84 \mathrm{mmol} / \mathrm{L}$, respectively, in the general Shenzhen population aged from 20 to 96 years.

There were significant differences in TG, HDL-C, and LDL-C concentrations between men and women, whereas the mean concentration of TC was similar in both genders $(P=0.083)$. In addition, we found that serum lipid concentrations were significantly positively (including: TC, TG, LDL-C) or negatively (HDL-C) correlated to diabetes, hypertension, body size, and age (Table 2).

\section{Prevalence of dyslipidemia components}

In this study, $31.88 \%$ of the study population showed at least one abnormal lipid parameter. When analyzing for dyslipidemia components, a hypercholesterolemia was found in $14.49 \%$, a hypertriglyceridemia in $16.14 \%$, an elevated levels of low density lipoprotein cholesterol in $12.13 \%$, and abnormally low high density lipoprotein

Table 2 Relationships between related factors and levels of TG, TC, HDL-C and LDL-C in serum samples (mmol/L)

\begin{tabular}{|c|c|c|c|c|c|c|c|c|c|c|c|c|}
\hline \multirow[t]{2}{*}{ Variables } & \multicolumn{3}{|l|}{$\mathrm{TC}$} & \multicolumn{3}{|l|}{ TG } & \multicolumn{3}{|c|}{$\mathrm{HDL}-\mathrm{C}$} & \multicolumn{3}{|c|}{ LDL-C } \\
\hline & $\mathrm{n}$ & Median & Range & $n$ & Median & Range & $n$ & Median & Range & $n$ & Median & Range \\
\hline \multicolumn{13}{|l|}{ Gender } \\
\hline Men & 885 & 5.09 & $0.94-14.58$ & 885 & $1.36^{* * *}$ & $0.34-21.83$ & 885 & $1.28^{* * *}$ & $0.41-5.24$ & 885 & $3.26^{* * *}$ & $0.43-7.06$ \\
\hline Women & 1110 & 4.95 & $0.86-13.35$ & 1110 & 1.12 & $0.25-10.56$ & 1110 & 1.47 & $0.62-4.51$ & 1110 & 3.07 & $0.87-7.38$ \\
\hline \multicolumn{13}{|l|}{ Diabetes } \\
\hline Yes & 175 & $5.18^{*}$ & $1.02-10.49$ & 175 & $1.56^{* * *}$ & $0.47-21.83$ & 175 & $1.25^{* * *}$ & $0.68-2.28$ & 175 & $3.32^{* *}$ & $1.30-6.50$ \\
\hline No & 1820 & 4.99 & $0.86-14.58$ & 1820 & 1.20 & $0.25-21.22$ & 1820 & 1.39 & $0.41-5.24$ & 1820 & 3.14 & $0.43-7.38$ \\
\hline \multicolumn{13}{|l|}{ Hypertension } \\
\hline Yes & 445 & $5.34^{* * *}$ & $1.49-13.35$ & 445 & $1.57^{* * *}$ & $0.47-11.47$ & 445 & $1.32^{* * *}$ & $0.60-2.41$ & 445 & $3.44^{* * *}$ & $1.06-7.38$ \\
\hline No & 1550 & 4.93 & $0.86-14.58$ & 1550 & 1.14 & $0.25-21.83$ & 1550 & 1.40 & $0.41-5.24$ & 1550 & 3.07 & $0.43-7.06$ \\
\hline \multicolumn{13}{|l|}{ Body size } \\
\hline Underweight & 123 & $4.51^{* * *}$ & $0.87-9.44$ & 123 & $0.93^{* * *}$ & $0.25-3.77$ & 123 & $1.56^{* * *}$ & $1.01-2.72$ & 123 & $2.63^{* * *}$ & $1.26-6.89$ \\
\hline Normal weight & 1027 & 4.89 & $0.86-14.58$ & 1027 & 1.05 & $0.27-21.83$ & 1027 & 1.46 & $0.41-5.24$ & 1027 & 3.03 & $0.43-7.38$ \\
\hline Overweight & 639 & 5.21 & $0.94-9.99$ & 639 & 1.52 & $0.41-20.38$ & 639 & 1.30 & $0.63-2.29$ & 639 & 3.36 & $1.06-6.99$ \\
\hline Obese & 206 & 5.31 & $1.49-9.14$ & 206 & 1.80 & $0.53-13.99$ & 206 & 1.23 & $0.60-2.06$ & 206 & 3.44 & $1.59-6.43$ \\
\hline \multicolumn{13}{|l|}{ Age groups (years) } \\
\hline $20-29$ & 269 & $4.51^{* * * *}$ & $0.86-9.57$ & 269 & $0.96^{* * *}$ & $0.33-13.99$ & 269 & $1.43^{* *}$ & $0.74-2.60$ & 269 & $2.68^{* * *}$ & $1.26-6.80$ \\
\hline $30-39$ & 545 & 4.80 & $0.87-14.58$ & 545 & 1.10 & $0.25-21.22$ & 545 & 1.37 & $0.74-5.24$ & 545 & 2.99 & $0.43-7.06$ \\
\hline $40-49$ & 434 & 5.07 & $1.02-9.44$ & 434 & 1.27 & $0.27-20.38$ & 434 & 1.34 & $0.41-2.66$ & 434 & 3.17 & $1.25-8.89$ \\
\hline $50-59$ & 316 & 5.42 & $0.94-9.31$ & 316 & 1.40 & $0.40-11.21$ & 316 & 1.41 & $0.63-2.71$ & 316 & 3.45 & $1.30-6.99$ \\
\hline $60-69$ & 251 & 5.40 & $5.40-10.49$ & 251 & 1.46 & $0.53-21.83$ & 251 & 1.37 & $0.62-4.51$ & 251 & 3.50 & $1.56-6.50$ \\
\hline $70-79$ & 150 & 5.28 & $1.36-13.35$ & 150 & 1.34 & $0.47-4.71$ & 150 & 1.35 & $0.83-2.31$ & 150 & 3.42 & $2.00-7.38$ \\
\hline$\geq 80$ & 30 & 5.46 & $2.77-7.94$ & $\mathrm{~m}$ & 1.20 & $0.48-7.36$ & 30 & 1.37 & $1.00-2.12$ & 30 & 3.33 & $1.06-6.18$ \\
\hline
\end{tabular}

*: $P<0.05 ; * *: P<0.01 ; * * *: P<0.001$

TC Total cholesterol, TG Triglycerides, HDL-C High density lipoprotein cholesterol, LDL-C Low density lipoprotein cholesterol 
cholesterol in $8.82 \%$ of the study participants (Table 3). Almost one sixths of the study participants (15.99\%) showed more than one abnormal lipid fractions, and in 4 subjects deranged levels of all four lipid fractions was found (data not shown).

The prevalence of dyslipidemia components according to gender, age, body size, and other characteristics is provided in Table 4. Higher hypertrigylceridemia prevalence was found among those men who were ever smoker, central obesity, overweight and obese, and older. The prevalence of high TC, high LDL-C, and low HDL-C was significantly high in those with abnormal body weight. The prevalence of high TC was significantly high among the respondents with alcohol drinking and the aged. The prevalence of low HDL-C was found to be significantly high in men, ever smoker, and those with central obesity, whereas the prevalence of high LDL-C was significantly associated with age.

\section{Prevalence and influencing factors of dyslipidemia}

Dyslipidemia was found for 636 (31.88 \%) of the participants. A positive history of dyslipidemia disease in the past year, and/or currently receiving treatment with lipid-lowering medications was described by 55 subjects, so that the total prevalence of dyslipidemia was $34.64 \%$. Adjusted for age on the basis of Shenzhen census 2010, the prevalence of dyslipidemia was $25.98 \%$. This represents 2.2 million Shenzhen adults aged from 20 to 96 years with dyslipidemia. Among subjects with dyslipidemia,
$173(25.04 \%)$ were aware of the diagnosis of their condition.

A number of potential independent factors that might influence the prevalence of dyslipidemia were evaluated by univariate logistic regression model. The results revealed that the variables of gender, diabetes, hypertension, central obesity, smoking status, body size, marital status, educational level, employment status, household per capita income (data not shown), and age were significantly associated with the prevalence of dyslipidemia (Table 5). On the contrary, the prevalence of dyslipidemia was found not to be significantly higher in nonhabitual drinkers and habitual drinkers, and exercisers. In order to further evaluate the main factors contributed to the prevalence of dyslipidemia and control potential confounding factors, multiple stepwise regression analyses were also performed. Stepwise multiple regression analysis revealed that the presence of dyslipidemia was still significantly associated with increasing age, diabetes, hypertension, body size, and smoking status (Table 6). With regard to health status, participants with diabetes had a 1.498 fold increased prevalence of dyslipidemia $(P=0.022)$, and participants with hypertension had 1.595 fold increased prevalence of dyslipidemia $(P<0.001)$. With regard to health behaviors, ever smokers more likely to had dyslipidemia $(\mathrm{OR}=2.033, P<0.001)$. Evaluation of body size suggested that, participants with high body size had a greater prevalence of dyslipidemia (overweight: $\mathrm{OR}=3.433, P<0.001$; obese: $\mathrm{OR}=4.405, P<$

Table 3 Proportion (95\% confidence interval) of concentrations classification of TC, TG, HDL-C and LDL-C in Shenzhen adults

\begin{tabular}{|c|c|c|c|}
\hline & \multicolumn{3}{|c|}{ Prevalence, \% (95 \% Cl) } \\
\hline & Overall & Men & Women \\
\hline \multicolumn{4}{|c|}{ Serum TC (mmol/L) } \\
\hline$<5.18$ & $56.19(54.0-58.4)$ & $53.22(49.9-56.5)$ & $58.56(55.6-61.4)$ \\
\hline $5.18-6.21$ & $29.32(27.4-31.3)$ & $33.00(29.9-36.1)$ & $26.39(23.9-29.0)$ \\
\hline$\geq 6.22$ & $14.49(13.0-16.1)$ & $13.78(11.6-16.2)$ & $15.05(13.0-17.2)$ \\
\hline \multicolumn{4}{|c|}{ Serum TG (mmol/L) } \\
\hline$<1.70$ & $70.33(68.3-72.3)$ & $63.28(60.1-66.4)$ & $75.95(73.4-78.4)$ \\
\hline $1.70-2.25$ & $13.53(12.1-15.1)$ & $14.91(12.7-17.4)$ & $12.43(10.6-14.5)$ \\
\hline$\geq 2.26$ & $16.14(14.6-17.8)$ & $21.81(19.2-24.6)$ & $11.62(9.8-13.6)$ \\
\hline \multicolumn{4}{|c|}{ Serum HDL-C (mmol/L) } \\
\hline$<1.04$ & $8.82(7.6-10.1)$ & $14.24(12.0-16.6)$ & $4.50(3.4-5.8)$ \\
\hline $1.04-1.54$ & $62.11(60.0-64.2)$ & $68.47(65.4-71.5)$ & $57.03(54.1-59.9)$ \\
\hline$\geq 1.55$ & $29.07(27.1-31.1)$ & 17.29 (14.9-19.9) & $38.47(35.6-41.4)$ \\
\hline \multicolumn{4}{|c|}{ Serum LDL-C (mmol/L) } \\
\hline$<3.37$ & $59.80(57.6-61.9)$ & $55.25(52.0-58.5)$ & $63.42(60.6-66.2)$ \\
\hline $3.37-4.13$ & $28.07(26.1-30.1)$ & $32.66(29.6-35.8)$ & $24.42(21.9-27.0)$ \\
\hline$\geq 4.14$ & $12.13(10.7-13.6)$ & $12.09(10.1-14.3)$ & $12.16(10.3-14.2)$ \\
\hline
\end{tabular}

TC Total cholesterol, TG Triglycerides, HDL-C High density lipoprotein cholesterol, LDL-C Low density lipoprotein cholesterol 
Table 4 The prevalence of dyslipidemia components

\begin{tabular}{|c|c|c|c|c|c|}
\hline \multirow[t]{2}{*}{ Characteristics } & \multirow[t]{2}{*}{$\mathrm{N}$} & High TC & \multirow{2}{*}{$\frac{\text { High TG }}{\mathrm{n}(\%)}$} & \multirow{2}{*}{$\frac{\text { Low HDL-C }}{\mathrm{n}(\%)}$} & \multirow{2}{*}{$\frac{\text { High LDL-C }}{\mathrm{n}(\%)}$} \\
\hline & & n (\%) & & & \\
\hline \multicolumn{6}{|l|}{ Gender } \\
\hline Men & 885 & $122(13.78)$ & $193(21.81)^{* * *}$ & $126(14.24)^{* * *}$ & $107(12.09)$ \\
\hline Women & 1110 & $167(15.05)$ & $129(11.62)$ & $50(4.50)$ & $135(12.16)$ \\
\hline \multicolumn{6}{|l|}{ Smoking status } \\
\hline Ever smoker & 414 & $65(15.70)$ & $109(26.33)^{* * *}$ & $66(15.94)^{* * *}$ & $52(12.56)$ \\
\hline Never smoker & 1581 & $224(14.17)$ & $213(13.47)$ & $110(6.95)$ & $190(12.02)$ \\
\hline \multicolumn{6}{|l|}{ Central obesity } \\
\hline Yes & 279 & $45(16.13)$ & $85(30.47)^{* * *}$ & $44(15.77)^{* * *}$ & $41(14.69)$ \\
\hline No & 1716 & $244(14.22)$ & $237(13.81)$ & $132(7.69)$ & $201(11.71)$ \\
\hline \multicolumn{6}{|l|}{ Drinking habit } \\
\hline Non-drinker & 1295 & $118(9.11)^{* *}$ & $203(15.68)$ & $109(8.42)$ & $165(12.74)$ \\
\hline Non-habitual drinker & 586 & $83(14.16)$ & $93(15.87)$ & $60(10.24)$ & 65 (11.09) \\
\hline Habitual drinker & 114 & $18(15.80)$ & $26(22.81)$ & $7(7.02)$ & $12(10.53)$ \\
\hline \multicolumn{6}{|l|}{ Body size } \\
\hline Underweight & 123 & $17(13.82)^{* *}$ & $2(1.63)^{* * *}$ & $1(0.81)^{* * *}$ & $13(10.57)^{* * *}$ \\
\hline Normal weight & 1027 & $118(11.49)$ & $93(9.06)$ & $50(4.87)$ & $87(8.47)$ \\
\hline Overweight & 639 & $118(18.47)$ & $158(23.62)$ & $84(13.15)$ & $111(17.37)$ \\
\hline Obese & 206 & $36(17.48)$ & $69(30.53)$ & $41(19.90)$ & $31(15.05)$ \\
\hline \multicolumn{6}{|l|}{ Age groups (years) } \\
\hline $20-29$ & 269 & $17(6.32)^{* * *}$ & $18(6.69)^{* * *}$ & $17(6.32)$ & $15(5.58)^{* * *}$ \\
\hline $30-39$ & 545 & $48(8.81)$ & 87 (15.96) & 55 (10.09) & 35 (6.42) \\
\hline $40-49$ & 434 & $51(11.75)$ & 75 (17.28) & 38 (8.76) & 43 (9.91) \\
\hline 50-59 & 316 & $73(23.10)$ & 61 (19.30) & $32(10.12)$ & 59 (18.67) \\
\hline $60-69$ & 251 & $57(22.71)$ & $55(21.91)$ & $23(9.16)$ & $52(20.63)$ \\
\hline 70-79 & 150 & $35(23.33)$ & $23(15.33)$ & $10(6.67)$ & 31 (20.67) \\
\hline$\geq 80$ & 30 & $8(26.67)$ & $3(10.00)$ & $1(3.33)$ & $7(23.33)$ \\
\hline
\end{tabular}

*: $P<0.05 ;{ }^{* *}: P<0.01 ;{ }^{* * *}: P<0.001$

TC Total cholesterol, TG Triglycerides, HDL-C High density lipoprotein cholesterol, LDL-C Low density lipoprotein cholesterol

0.001). Compared with participants 20 to 29 years of age, those who were 50 to 59 years of age had a greater prevalence of dyslipidemia $(\mathrm{OR}=3.012, P<0.001)$.

\section{Discussions}

With economic growth and adverse changes in lifestyle (such as a high intake of dietary saturated fat, physical inactivity and smoking), cardiovascular diseases have become the leading cause of death in China [20]. Dyslipidemia is one of the most important independent modifiable risk factors for cardiovascular diseases. A prevention measure against the epidemic of dyslipidemia will undoubtedly depress the morbidity and mortality of cardiovascular diseases.

This study was conducted in 8 districts of Shenzhen to comprehensive assess the prevalence of dyslipidemia as well as the associated risk factors. Our results indicated that the prevalence of high TC, high TG, high LDL-C, and low HDL-C were $14.49 \%, 16.14 \%, 12.13 \%$, and
$8,82 \%$, respectively. It was also revealed that the prevalence of dyslipidemia was about 34.64 \% (standardized rate per 2010 Shenzhen population: 25.98 \%). Furthermore, our results suggested that the presence of dyslipidemia was significantly associated with age, diabetes, hypertension, body size, and smoking status.

Several previous epidemiological studies reported serum lipid concentrations in Chinese populations [9, 21, 22]. In those studies, the China National Diabetes and Metabolic Disorders Study measured serum lipids in a nationally representative sample of 47,352 Chinese aged 20 years or above and provided the best comparison data for our study [22]. When compared with the findings from the China National Diabetes and Metabolic Disorders Study, the mean levels of TC $(5.11 \mathrm{mmol} / \mathrm{L}$ vs $4.72 \mathrm{mmol} / \mathrm{L})$, TG (1.59 $\mathrm{mmol} / \mathrm{L}$ vs $1.57 \mathrm{mmol} / \mathrm{L}), \mathrm{HDL}-\mathrm{C}(1.42 \mathrm{mmol} / \mathrm{L}$ vs $1.30 \mathrm{mmol} / \mathrm{L})$, and LDL-C (3.22 mmol/L vs $2.68 \mathrm{mmol} / \mathrm{L})$ in this study did subtle changes [22]. Also other regional 
Table 5 Univariate logistic regression analysis of sociodemographic and lifestyle factors associated with dyslipidemia

\begin{tabular}{|c|c|c|}
\hline Characteristics & OR $(95 \% \mathrm{Cl})$ & $P$ value \\
\hline Gender & $1.719(1.427-2.070)$ & $<0.001$ \\
\hline Diabetes & $2.502(1.829-3.423)$ & $<0.001$ \\
\hline Hypertension & $2.818(2.271-3.498)$ & $<0.001$ \\
\hline Central obesity & $2.280(1.765-2.944)$ & $<0.001$ \\
\hline Physical activity & $1.057(0.841-1.328)$ & 0.634 \\
\hline Smoking status & $1.923(1.543-2.397)$ & $<0.001$ \\
\hline \multicolumn{3}{|l|}{ Drinking habit } \\
\hline Non-drinker & 1 (reference) & \\
\hline Non-habitual drinker & $1.013(0.825-1.244)$ & 0.901 \\
\hline Habitual drinker & $1.025(0.686-1.532)$ & 0.902 \\
\hline \multicolumn{3}{|l|}{ Body size } \\
\hline Underweight & 1 (reference) & \\
\hline Normal weight & $1.613(0.979-2.660)$ & 0.061 \\
\hline Overweight & 4.914 (2.970-8.129) & $<0.001$ \\
\hline Obese & $6.382(3.674-11.084)$ & $<0.001$ \\
\hline \multicolumn{3}{|l|}{ Marital status } \\
\hline Single & 1 (reference) & \\
\hline Married & $2.139(1.376-3.325)$ & 0.001 \\
\hline Divorced & $2.641(1.065-6.551)$ & 0.036 \\
\hline Widowed & 3.741 (1.980-7.069) & $<0.001$ \\
\hline \multicolumn{3}{|l|}{ Educational level } \\
\hline$\geq 12$ years & 1 (reference) & \\
\hline 10-12 years & $0.974(0.728-1.304)$ & 0.860 \\
\hline 7-9 years & $1.417(1.042-1.928)$ & 0.026 \\
\hline $1-6$ years & $1.557(1.094-2.217)$ & 0.014 \\
\hline 0 year & $2.064(1.222-3.487)$ & 0.007 \\
\hline \multicolumn{3}{|l|}{ Employment status } \\
\hline Employed & 1 (reference) & \\
\hline Homemaker & $1.263(0.958-1.666)$ & 0.098 \\
\hline Others & $1.390(0.976-1.981)$ & 0.068 \\
\hline Retired & $2.074(1.655-2.599)$ & $<0.001$ \\
\hline Unemployed & $0.901(0.558-1.456)$ & 0.671 \\
\hline \multicolumn{3}{|l|}{ Age groups (years) } \\
\hline $20-29$ & 1 (reference) & $<0.001$ \\
\hline $30-39$ & $1.944(1.346-2.808)$ & $<0.001$ \\
\hline $40-49$ & $2.333(1.602-3.396)$ & $<0.001$ \\
\hline $50-59$ & $4.325(2.938-6.367)$ & $<0.001$ \\
\hline $60-69$ & $4.301(2.875-6.434)$ & $<0.001$ \\
\hline $70-79$ & $4.020(2.559-6.316)$ & $<0.001$ \\
\hline$\geq 80$ & 3.707 (1.685-8.159) & 0.001 \\
\hline
\end{tabular}

Table 6 Multivariate stepwise logistic regression analysis of factors associated with dyslipidemia

\begin{tabular}{|c|c|c|}
\hline Characteristics & OR (95 \% Cl) & $P$ value \\
\hline \multicolumn{3}{|l|}{ Diabetes } \\
\hline No & 1 (reference) & \\
\hline Yes & $1.498(1.061-2.114)$ & 0.022 \\
\hline \multicolumn{3}{|l|}{ Hypertension } \\
\hline No & 1 (reference) & \\
\hline Yes & $1.595(1.234-2.060)$ & $<0.001$ \\
\hline \multicolumn{3}{|l|}{ Smoking status } \\
\hline Ever smoker & 1 (reference) & \\
\hline Never smoker & $2.033(1.604-2.577)$ & $<0.001$ \\
\hline \multicolumn{3}{|l|}{ Body size } \\
\hline Underweight & 1 (reference) & \\
\hline Normal weight & $1.280(0.763-2.147)$ & 0.351 \\
\hline Overweight & $3.433(2.033-5.798)$ & $<0.001$ \\
\hline Obese & $4.045(2.267-7.215)$ & $<0.001$ \\
\hline \multicolumn{3}{|l|}{ Age groups (years) } \\
\hline $20-29$ & 1 (reference) & \\
\hline $30-39$ & $1.747(1.189-2.567)$ & 0.004 \\
\hline $40-49$ & $1.761(1.184-2.618)$ & 0.005 \\
\hline $50-59$ & $3.012(1.989-4.563)$ & $<0.001$ \\
\hline $60-69$ & $2.880(1.860-4.460)$ & $<0.001$ \\
\hline 70-79 & $2.722(1.657-4.471)$ & $<0.001$ \\
\hline$\geq 80$ & $2.280(0.974-5.339)$ & 0.058 \\
\hline
\end{tabular}

studies have previously examined serum lipid levels in local residents. Wang and co-researchers reported that the mean levels of TC, TG, HDL-C, and LDL-C in Beijing were $5.05 \mathrm{mmol} / \mathrm{L}, 1.53 \mathrm{mmol} / \mathrm{L}, 1.50 \mathrm{mmol} / \mathrm{L}$, and $3.27 \mathrm{mmol} / \mathrm{L}$, respectively [23]. Wu et al. reported that the mean levels of TC, TG, and HDL-C in Shanghai were $4.70 \mathrm{mmol} / \mathrm{L}, 1.50 \mathrm{mmol} / \mathrm{L}$, and $1.40 \mathrm{mmol} / \mathrm{L}$, respectively [24]. Our results were similar to those of the above mentioned studies.

During the past several decades, favorable trends in lipid levels have occurred among adults in the United States [25]. For example, the mean levels of TC declined from 206 to $196 \mathrm{mg} / \mathrm{dL}$ between 1988 to 1994 and 2007 to 2010. Additionally, the mean concentrations of LDL-C declined from 129 to $116 \mathrm{mg} / \mathrm{dL}$ during 1988-1994 and 2007-2010. On the contrary, compared to the result from the 2002 China National Nutrition and Health Survey, unfavorable trends in lipid levels have occurred among adults in Shenzhen [26]. It is in consistent with the general increase in the mean levels of serum lipid (including: TC, TG, LDL-C) in China with the increasing urbanization and changes in lifestyle [22]. These findings showed important public health implications, which might explain the recent 
rapid increase in cardiovascular disease burdens in Shenzhen [13].

The prevalence of high TC (14.49\%) and high LDL-C $(12.13 \%)$ found in this study were significantly higher than average Chinese national standards $(9.0 \%$ and $6.5 \%$, respectively), whereas the prevalence of low HDL$\mathrm{C}(8.82 \%)$ was significantly lower than the national average $(22.30 \%)$ [22]. High TC is one of the common lipid disorders in western countries. With the economic growth and the increasing popularity of western foods in Shenzhen, an increasing number of people have changed their dietary patterns. For example, they may choose to consume a more westernized diet, which is high in fat and cholesterol. Therefore, it may be the main contributor to the high TC in Shenzhen. Compared with studies carried out in some other regions, the prevalence of dyslipidemia components in Shenzhen were lower than those in Shanghai [24], Delhi [14], and Turkish [1], but a higher prevalence of high TC or high TG, and lower prevalence of high LDL-C or low HDL-C were found in Shenzhen than in Shaoxing [12]. The reported prevalence of dyslipidemia components varied among Shenzhen, Shanghai, Delhi, Turkish and Shaoxing, depending on the information available for the given population and the diagnostic criteria used. In the present study, the most prevalent component of dyslipidemia was the increased TG with a prevalence of $16.14 \%$. A similar study reported that the most prominent change in serum lipids in Beijing and Guangzhou over the past 20 years was the increase in serum TG levels, which were dramatically influenced by the increased consumption of diets high in simple carbohydrates and fat [27]. However, other studies found that the major types of dyslipidemia in China and other Asian countries are low HDL-C $[24,28,29]$, whereas high TC as the most common lipid disorder in Western countries [1].

The prevalence of dyslipidemia in our study was partially comparable to figures reported in previous epidemiological studies [10, 12, 24]. For example, in a cross sectional study with a representative sample of 5,761 Chinese adults aged from 18 to 79 years, $35.40 \%$ of the subjects had dyslipidemia [10]. In addition, in a recent study on 11,877 Shaoxing residents aged 20 years or older, the age standardized prevalence of dyslipidemia was $25.80 \%$ (the age standardized dyslipidemia prevalence in Shenzhen was $25.98 \%$ ) [12]. Our results, together with other study, confirmed that the prevalence of dyslipidemia is increasing in Shenzhen [22, 26]. Notably, the rate of awareness documented in this survey is lower than those recently reported from cross sectional study conducted in Beijing, and elsewhere during the same periods [30, 31]. It is obviously that Shenzhen is going to experience a large increase in the prevalence of cardiovascular disease, if reasonable and effective control measures not taken.
Previous studies reported that age did appear to influence dyslipidemia risk $[1,11,12,14,24]$. In the present study, compared with participants aged from 20 to 29 years, those who were 50 to 59 years of age had a greater prevalence of dyslipidemia. With the aging population trend in China, the picture of the public health posed by dyslipidemia will be aggravated. However, the precise mechanisms of the effect of age on serum lipid concentrations are not exactly known-they may be related to hereditary characteristics and degenerative processes as well as creeping weight gain and progressive development of insulin resistance [1]. In addition, the prevalence of dyslipidemia increased with BMI in our study, which was consistent with some previous studies $[1,14,32,33]$. The lipid abnormalities in overweight and obesity is likely a consequence of insulin resistance [34]. The overweight and obesity prevalence in 2010 was $30.6 \%$ and $12.0 \%$, which have increased by $34.21 \%$ and $69.01 \%$, respectively, since 2002 [35, 36]. By comparison, we found that the prevalence of abnormal body weight in Shenzhen was comparable with that in mainland China as a whole [35]. The rapid development of overweight or obesity in Shenzhen will undoubtedly accelerate the prevalence of dyslipidemia.

Several well established risk factors for dyslipidemia, including hypertension and diabetes have been evaluated $[1,12,14,33,37]$. We found a consistent pattern of an increasing prevalence of dyslipidemia with increasing fasting blood glucose, and blood pressure. Several factors are likely to be responsible for diabetic dyslipidemia: insulin effects on liver apoprotein production, regulation of lipoprotein lipase, actions of cholesteryl ester transfer protein, and peripheral actions of insulin on adipose and muscle [38]. Previous studies have demonstrated that genetic factors may influence development of dyslipidemia hypertension, nongenetic, potentially modifiable aspects of obesity are also closely related to expression of this clinically important syndrome [39]. Given the relative high prevalence of dyslipidemia in patients with hypertension or diabetes, it is imperative that any patient with hypertension or diabetes be screened for dyslipidemia.

Other researchers observed associations between dyslipidemia and smoking [11, 40, 41]. Our results also showed that smoking status of the participants was related to the prevalence of dyslipidemia. Concentrations of TG in current smokers were shown to be significantly higher and concentrations of HDL-C lower than those who had never smoked or had stopped smoking [41]. Chitra et al. reported the presence of carbon monoxide and nicotine content in the cigarette smoke, which decreases the activity of lipoprotein lipase resulted in the elevated levels of triglycerides [42].

Potential limitations of our study should be mentioned. First, no dietary intake was measured. Therefore, 
our study cannot show their effect on serum lipids. Second, potential recalled sources of bias including recall bias and self-reported information. Finally, other risk factors (such as stress, genetic factors) were not taken into account.

\section{Conclusions}

Dyslipidemia was present in about $34.64 \%$ of the study population in Shenzhen, and the awareness rate of dyslipidemia was $25.04 \%$. Risk factors associated with dyslipidemia were aging, higher body size, hypertension, diabetes, and smoking. Dyslipidemia is becoming an important public health problem. Obesity can be used to screen for dyslipidemia along with other coexisting risk factors such as hypertension and diabetes. There is an urgent need to institute more positive public health measures and screening programs and to treat patients diagnosed with dyslipidemia.

\section{Competing interests}

The authors declare that they have no competing interests.

\section{Authors' contributions}

WN and HC conceived and designed the experiment, ZZ and JS performed the experiments, $X Y$ analysed the data, $X L$ and JX contributed reagents/ materials/analysis tools, WN wrote the manuscript. All authors read and approved the final manuscript.

\section{Acknowledgments}

We are grateful to all the volunteers for participating in the present study, and to the staff of these institutions (District center for chronic disease control) who contributed to this research. We are also grateful to the China Health and Nutrition Survey Program. This study was supported by the National Key Technology Research and Development Program of the Ministry of Science and Technology of China (Grant No. 2012BAI02B02).

Received: 10 March 2015 Accepted: 3 July 2015

Published online: 14 July 2015

\section{References}

1. Bayram F, Kocer D, Gundogan K, Kaya A, Demir O, Coskun R, et al. Prevalence of dyslipidemia and associated risk factors in Turkish adults. J Clin Lipidol. 2014;8(2):206-16.

2. Comanor WS, Scherer FM. Mergers and innovation in the pharmaceutical industry. J Health Econ. 2013;32(1):106-13.

3. Fuentes R, Uusitalo T, Puska P, Tuomilehto J, Nissinen A. Blood cholesterol level and prevalence of hypercholesterolaemia in developing countries: a review of population-based studies carried out from 1979 to 2002. Eur J Cardiovasc Prev Rehabil. 2003;10(6):411-9.

4. Murray CJ, Lopez AD. Mortality by cause for eight regions of the world: Global Burden of Disease Study. Lancet. 1997;349(9061):1269-76.

5. Moran A, Gu D, Zhao D, Coxson P, Wang YC, Chen CS, et al. Future cardiovascular disease in china: markov model and risk factor scenario projections from the coronary heart disease policy model-china. Circ Cardiovasc Qual Outcomes. 2010;3(3):243-52.

6. Janus ED, Tideman PA, Dunbar JA, Kilkkinen A, Bunker SJ, Philpot B, et al. Dyslipidaemia in rural Australia: prevalence, awareness, and adherence to treatment guidelines in the Greater Green Triangle Risk Factor Study. Med J Aust. 2010;192(3):127-32.

7. Goff Jr DC, Bertoni AG, Kramer H, Bonds D, Blumenthal RS, Tsai MY, et al. Dyslipidemia prevalence, treatment, and control in the Multi-Ethnic Study of Atherosclerosis (MESA): gender, ethnicity, and coronary artery calcium. Circulation. 2006;113(5):647-56.

8. Grundy SM, Cleeman Jl, Merz CN, Brewer Jr HB, Clark LT, Hunninghake DB, et al. Implications of recent clinical trials for the National Cholesterol
Education Program Adult Treatment Panel III Guidelines. J Am Coll Cardiol. 2004:44(3):720-32.

9. He J, Gu D, Reynolds K, Wu X, Muntner P, Zhao J, et al. Serum total and lipoprotein cholesterol levels and awareness, treatment, and control of hypercholesterolemia in China. Circulation. 2004;110(4):405-11.

10. Cai L, Zhang L, Liu A, Li S, Wang P. Prevalence, awareness, treatment, and control of dyslipidemia among adults in Beijing, China. J Atheroscler Thromb. 2011;19(2):159-68.

11. Wang S, Xu L, Jonas JB, You QS, Wang YX, Yang H. Prevalence and associated factors of dyslipidemia in the adult Chinese population. PLoS One. 2011;6(3), e17326

12. Shi $L, H u$ J, Zhu K, Fu Y, Xia R, Hu X. Changes of prevalence of dyslipidemia among adults: a cross-sectional study with a 2-year follow-up in urban southeast China. Clin Lipidol. 2014;9(1):33-47.

13. Zhang D, Mou J, Cheng J, Griffiths S. Public health services in Shenzhen: a case study. Public Health. 2011;125(1):15-9.

14. Sharma U, Kishore J, Garg A, Anand T, Chakraborty M, Lali P. Dyslipidemia and associated risk factors in a resettlement colony of Delhi. J Clin Lipidol. 2013;7(6):653-60.

15. Stolt P, Bengtsson C, Nordmark B, Lindblad S, Lundberg I, Klareskog $L$, et al. Quantification of the influence of cigarette smoking on rheumatoid arthritis: results from a population based case-control study, using incident cases. Ann Rheum Dis. 2003:62(9):835-41.

16. Zhang L, Wang F, Wang L, Wang W, Liu B, Liu J, et al. Prevalence of chronic kidney disease in China: a cross-sectional survey. Lancet. 2012;379(9818):815-22.

17. Kantomaa MT, Stamatakis E, Kankaanpää A, Kaakinen M, Rodriguez A, Taanila A, et al. Physical activity and obesity mediate the association between childhood motor function and adolescents' academic achievement. Proc Natl Acad Sci U S A. 2013;110(5):1917-22.

18. Committee CADP. China Adult Dyslipidemia Prevention Guide. Beijing: People's Health Publishing House; 2007.

19. Wu Y, Huxley R, Li L, Anna V, Xie G, Yao C, et al. Prevalence, awareness, treatment, and control of hypertension in China data from the China National Nutrition and Health Survey 2002. Circulation. 2008;118(25):2679-86.

20. He J, Gu D, Wu X, Reynolds K, Duan X, Yao C, et al. Major causes of death among men and women in China. N Engl J Med. 2005;353(11):1124-34.

21. Li Y, Li Y, Davis C, Chen Z, Tao S, Folsom A, et al. Serum cholesterol changes from 1983-1984 to 1993-1994 in the People's Republic of China. Nutr Metab Cardiovasc Dis. 2002;12(3):118-26.

22. Yang $W$, Xiao J, Yang Z, Ji L, Jia W, Weng J, et al. Serum lipids and lipoproteins in Chinese men and women. Circulation. 2012;125(18):2212-21.

23. Cai L, Zhang L, Liu A, Li S, Wang P. Prevalence, awareness, treatment, and control of dyslipidemia among adults in Beijing, China. J Atheroscler Thromb. 2012;19(2):159-68.

24. Wu J-Y, Duan X-Y, Li L, Dai F, Li Y-Y, Li X-J, et al. Dyslipidemia in Shanghai, China. Pre Med. 2010;51(5):412-5.

25. Carroll MD, Kit BK, Lacher DA, Shero ST, Mussolino ME. Trends in lipids and lipoproteins in US adults, 1988-2010. JAMA. 2012;308(15):1545-54.

26. Zhao WH, Zhang J, Zhai Y, You Y, Man QQ, Wang CR, et al. Blood lipid profile and prevalence of dyslipidemia in Chinese adults. Biomed Environ Sci. 2007:20(4):329-35.

27. Zhou B, Rao X, Dennis BH, Li Y, Zhuo Q, Folsom AR, et al. The relationship between dietary factors and serum lipids in Chinese urban and rural populations of Beijing and Guangzhou. Int J Epidemiol. 1995;24(3):528-34.

28. Joshi SR, Anjana RM, Deepa M, Pradeepa R, Bhansali A, Dhandania VK, et al Prevalence of Dyslipidemia in Urban and Rural India: The ICMR-INDIAB Study. Plos One. 2014;9(5), e96808.

29. Stewart CP, Christian P, Wu LS, LeClerq SC, Khatry SK, West Jr KP. Prevalence and risk factors of elevated blood pressure, overweight, and dyslipidemia in adolescent and young adults in rural Nepal. Metab Syndr Relat Disord. 2013;11(5):319-28.

30. Li Z, Yang R, Xu G, Xia T. Serum lipid concentrations and prevalence of dyslipidemia in a large professional population in Beijing. Clin Chem. 2005:51(1):144-50.

31. Luo J-y, Ma Y-T, Yu Z-x, Yang Y-N, Xie X, Ma X. Prevalence, awareness, treatment and control of dyslipidemia among adults in Northwestern China: the cardiovascular risk survey. Lipids Health Dis. 2014;13(1):4.

32. Shen $\mathrm{Z}$, Munker $\mathrm{S}$, Wang $\mathrm{C}, \mathrm{Xu} \mathrm{L}, \mathrm{Ye} \mathrm{H}$, Chen $\mathrm{H}$, et al. Association between alcohol intake, overweight, and serum lipid levels and the risk analysis associated with the development of dyslipidemia. J Clin Lipidol. 2014;8(3):273-8 
33. Kerimkulova AS, Mirrakhimov AE, Nabiev MP, Neronova KV, Bayramukova AA, Alibaeva NT, et al. The association of leptin with dyslipidemia, arterial hypertension and obesity in Kyrgyz (Central Asian nation) population. BMC Res Notes. 2014;7(1):411.

34. Houston MC, Basile J, Bestermann WH, Egan B, Lackland D, Hawkins RG, et al. Addressing the global cardiovascular risk of hypertension, dyslipidemia, and insulin resistance in the southeastern United States. Am J Med Sci. 2005:329(6):276-91.

35. Chen C. Overview of obesity in Mainland China. Obes Rev. 2008;9(s1):14-21.

36. Li XY, Jiang Y, Hu N, Li YC, Zhang M, Huang ZJ, et al. Prevalence and characteristic of overweight and obesity among adults in China, 2010. Zhonghua Yu Fang Yi Xue Za Zhi. 2012;46(8):683-6.

37. Haffner SM. Management of dyslipidemia in adults with diabetes. Diabetes care. 1998;21(1):160-78.

38. Goldberg IJ. Diabetic dyslipidemia: causes and consequences. J Clin Endocrinol Metab. 2001;86(3):965-71.

39. Selby JV, Newman B, Quiroga J, Christian JC, Austin MA, Fabsitz RR Concordance for dyslipidemic hypertension in male twins. JAMA. 1991;265(16):2079-84.

40. Lee MH, Ahn SV, Hur NW, Choi DP, Kim HC, Suh I. Gender differences in the association between smoking and dyslipidemia: 2005 Korean National Health and Nutrition Examination Survey. Clin Chim Acta. 2011;412(17):1600-5

41. Tan X, Jiao G, Ren Y, Gao X, Ding Y, Wang X, et al. Relationship between smoking and dyslipidemia in western Chinese elderly males. J Clin Lab Anal. 2008:22(3):159-63.

42. Chitra S, Semmalar R, Devi CS. Effect of fish oil on cigarette smoking induced dyslipidemia in rats, Indian J Pharmacol. 2000:32(2):114-9.

\section{Submit your next manuscript to BioMed Central and take full advantage of:}

- Convenient online submission

- Thorough peer review

- No space constraints or color figure charges

- Immediate publication on acceptance

- Inclusion in PubMed, CAS, Scopus and Google Scholar

- Research which is freely available for redistribution 